\title{
Analysis, Design and Optimization of Multilayer Antenna Using Wave Concept Iterative Proces
}

\author{
Amri Houda* \\ Mohamed Cherif Messaadia University, Souk Ahras, Algeria \\ Actives Devices and Materials Laboratory, Larbi Ben M'Hidi University, Oum El Bouaghie, Algeria \\ ${ }^{*}$ Coressponding author, e-mail: amrihouda@gmail.com
}

\begin{abstract}
The wave concept iterative process is a procedure used for analyses a planar circuits This method consists in generating a recursive relationship between a wave source and reflected waves from the discontinuity plane which is divided into cells. A high computational speed has been achieved by using Fast Modal Transform (FMT). In this paper we study a patch antenna and multilayer circuits, to determine the electromagnetic characteristics of these structures.
\end{abstract}

Keywords: WCIP, planar circuit, patch antenna, multilayer circuit, FMT

\section{Introduction}

Antennas are a very important component of communication systems. By definition, an antenna is a device used to transform an RF signal, traveling on a conductor, into an electromagnetic wave in free space. Antennas demonstrate a property known as reciprocity, which means that an antenna will maintain the same characteristics regardless if it is transmitting or receiving. Most antennas are resonant devices, which operate efficiently over a relatively narrow frequency band. An antenna must be tuned to the same frequency band of the radio system to which it is connected; otherwise the reception and the transmission will be impaired. When a signal is fed into an antenna, the antenna will emit radiation distributed in space in a certain way.

\section{The Theoretical Basis}

\subsection{Definition of the WCIP}

WCIP method is based on full wave transverse wave formulation and the collection at the interfaces. A multiple reflection procedure is started using initial conditions and stopped once convergence is archived. Two operators relating incident waves and scattered waves in the spatial domain and in the spectral domain governs the scattering operator $\hat{S}$ and the reflection operator $\hat{\Gamma}$.

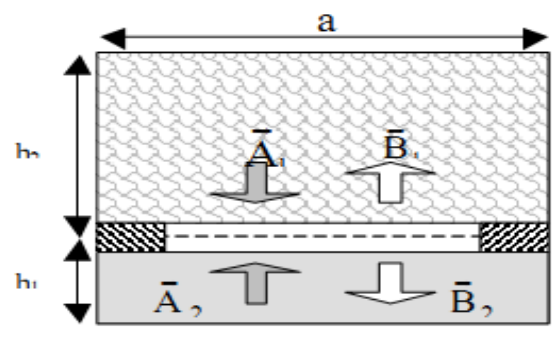

face view

Figure 1. The process Iterative [1] 
The wave concept iterative process WCIP is defined as [2-3].

$$
\begin{aligned}
\vec{A} & =\hat{S} \vec{B}+\vec{A}_{0} \\
\vec{B} & =\hat{\Gamma} \vec{A}
\end{aligned}
$$

Where the incident waves $\vec{A}$ and the scatered waves $\vec{B}$ are calculated from the tangentiel electric and magnetic fields $\vec{E}$ and $\vec{H}$ :

$$
\begin{aligned}
& \vec{A}_{i}=\frac{1}{2 \sqrt{Z_{0 i}}}\left(\vec{E}_{i}+Z_{0 i} \vec{J}_{i}\right) \\
& \vec{B}_{i}=\frac{1}{2 \sqrt{Z_{0 i}}}\left(\vec{E}_{i}-Z_{0 i} \vec{J}_{i}\right)
\end{aligned}
$$

where: $Z_{0 i}$ is the characteristic impedance of the same medium

$i$ indicates the medium 1 or 2

$\vec{J}_{i}=\vec{n} \wedge \vec{H}_{i}$ is the density of courant

\subsection{The Reflection Operator $\hat{\Gamma}$}

The reflection operator is deduced from the admittance operator and the wave's definition in (3)

$$
\begin{aligned}
& \Gamma_{m n}^{\alpha}=\sum_{m n}\left\langle f_{m n}^{\alpha}\left|\frac{1-Z_{0 i} Y_{m n}^{\alpha}}{1+Z_{0 i} Y_{m n}^{\alpha}}\right| f_{m n}^{\alpha}\right\rangle \\
& Y_{m n}^{T E}\left(\varepsilon_{r}\right)=\frac{\gamma_{m n}\left(\varepsilon_{r}\right)}{j \omega \mu_{0}} \\
& Y_{m n}^{T M}\left(\varepsilon_{r}\right)=\frac{j \omega \varepsilon_{0} \varepsilon_{r}}{\gamma_{m n}\left(\varepsilon_{r}\right)}
\end{aligned}
$$

\subsection{The Scattering (Diffraction) Operator $\widehat{S}$}

The scattering operator $\widehat{S}$ is deduced from the equivalent circuit on each sub domain of the interface $\mathrm{S}$. the boundary conditions is expressed for fields in the next equation:

$$
\begin{aligned}
& \vec{E}_{1}=\vec{E}_{2}=\overrightarrow{0} \quad \text { on the metal } \\
& \vec{E}_{1}=\vec{E}_{2} \neq \overrightarrow{0} \text { and } \vec{J}_{1}+\vec{J}_{2}=\overrightarrow{0} \text { on the isolante } \\
& \vec{E}_{1}=\vec{E}_{0}-Z_{0}\left(\vec{J}_{1}+\vec{J}_{2}\right)=\overrightarrow{0} \text { on the source }
\end{aligned}
$$

Moreover, since operators $(\hat{\Gamma}$ and $\hat{S})$ are defined in both the space and the spectral domains, the iterative process implies switching between these domains, which is ensured by the fast mode transform (FMT) and its inverse.

\subsection{Definition of Fast Modal Transformation FMT in Periodic Wave Guide}

The classic 2D Fast Fourier Transformation of the waves $A_{i x}, A_{i y}$ is defined as following: 


$$
\begin{aligned}
& \left|A_{x}(x, y)\right\rangle=\sum_{m n} A_{x m n}\left|e^{-j \beta_{x m} x} e^{-j \beta_{n y} y}\right\rangle \\
& \left|A_{y}(x, y)\right\rangle=\sum_{m n} A_{y m n}\left|e^{-j \beta_{x x} x} e^{-j \beta_{n y} y}\right\rangle \\
& \beta_{x m}=\frac{2 m \pi}{a} \quad \beta_{y m}=\frac{2 m \pi}{b}
\end{aligned}
$$

Where $\beta_{x m}=\frac{2 m \pi}{a} \quad \beta_{y m}=\frac{2 m \pi}{b}$ are the Fourier coefficients

We project the vectors $A(x, y)$ in the complete set of modes corresponding to rectangular periodic wave guide $\left|f^{\alpha}\right\rangle$.

$$
\begin{aligned}
& \left|A_{x}(x, y)\right\rangle=\sum_{m n} A_{x m n}^{T E}\left|f_{m n, x}^{T E}\right\rangle+\sum_{m n} A_{x m n}^{T M}\left|f_{m n, x}^{T M}\right\rangle \\
& \left|A_{y}(x, y)\right\rangle=\sum_{m n} A_{y m n}^{T E}\left|f_{m n, y}^{T E}\right\rangle+\sum_{m n} A_{y m n}^{T M}\left|f_{m n, y}^{T M}\right\rangle
\end{aligned}
$$

Projecting the equation (6) in the set of modes, we can deduce [4]:

$$
\begin{aligned}
& \sum_{m n} A_{m n}^{T E}=\left\langle f_{m n, x}^{T E} \mid A_{x}(x, y)\right\rangle+\left\langle f_{m n, y}^{T E} \mid A_{y}(x, y)\right\rangle \\
& \sum_{m n} A_{m n}^{T M}=\left\langle f_{m n, x}^{T M} \mid A_{x}(x, y)\right\rangle+\left\langle f_{m n, y}^{T M} \mid A_{y}(x, y)\right\rangle
\end{aligned}
$$

The multilayered structure can be modulated by an equivalent circuit illustrated in Figure 2 The two network port represents the dielectric between the conductor in the planes $\pi_{1}$ and $\pi_{2}$. $Y_{1}$ : is the admittance of upper metal box in the plan $\pi_{1}$ $Y_{3}$ : is the admittance of low metal box in the plan $\pi_{2}$

According to Figure 2 it is possible to establish the following equations
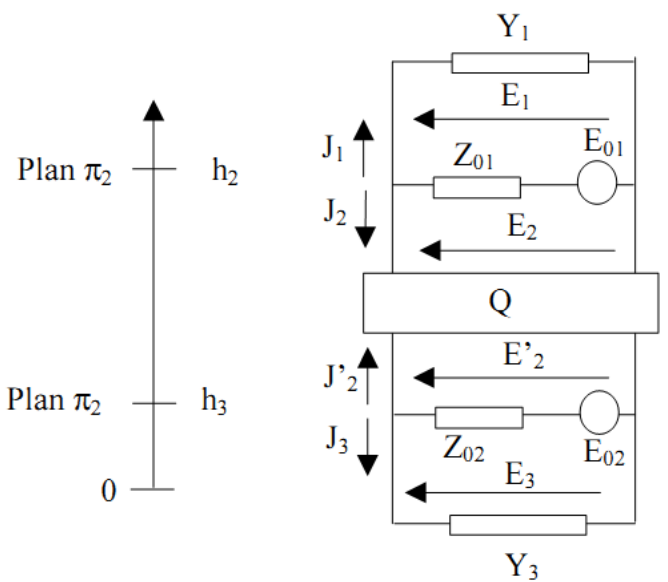

Figure 2. Equivalent circuit 
- Space domain:

$$
\begin{aligned}
& {\left[\begin{array}{l}
\vec{A}_{1} \\
\vec{A}_{2}
\end{array}\right]=S_{12}\left[\begin{array}{l}
\vec{B}_{1} \\
\vec{B}_{2}
\end{array}\right]+\left[\begin{array}{l}
\vec{A}_{01} \\
\vec{A}_{02}
\end{array}\right]} \\
& {\left[\begin{array}{l}
\vec{A}_{2}^{\prime} \\
\vec{A}_{3}
\end{array}\right]=S_{23}\left[\begin{array}{l}
\vec{B}_{2}^{\prime} \\
\vec{B}_{3}
\end{array}\right]+\left[\begin{array}{l}
\vec{A}_{02}^{\prime} \\
\vec{A}_{03}
\end{array}\right]}
\end{aligned}
$$

$S_{12}$ and $S_{23}$ have the same experience a $\hat{S}$

- Spectral domain:

$$
\begin{aligned}
& {\left[\begin{array}{l}
\vec{B}_{1} \\
\vec{B}_{3}
\end{array}\right]=\left[\begin{array}{cc}
\Gamma_{1}^{\alpha} & 0 \\
0 & \Gamma_{3}^{\alpha}
\end{array}\right]\left[\begin{array}{l}
\vec{A}_{1} \\
\vec{A}_{3}
\end{array}\right]} \\
& {\left[\begin{array}{l}
\vec{B}_{2} \\
\vec{B}_{2}^{\prime}
\end{array}\right]=\Gamma_{Q}\left[\begin{array}{l}
\vec{A}_{2} \\
\vec{A}_{2}^{\prime}
\end{array}\right]}
\end{aligned}
$$

$\Gamma_{1}^{\alpha}, \Gamma_{3}^{\alpha}$ : reflected operator in the spectral domain

$\Gamma_{Q}$ : is the admittance operator of network port

The $Q$ two ports network can be characterized by referring to the theory of the transmission lines [5].

\section{Modelisation and Results}

The new formulation of the iterative method is applied to two different structures: a patch antenna and multilayered structure.

\subsection{Simulation of Patch Antenna}

The proposed approach is first checked by considering the structure given in Figure 3 . This structure consists of a patch antenna connected to a microstrip line section. The box size is $35^{*} 64 \mathrm{~mm}$. The substrate characteristics are $\varepsilon=4.78$ with a thickness of $0.79 \mathrm{~mm}$. The line is 20 $\mathrm{mm}$ long and $2.34 \mathrm{~mm}$ wide, and the patch is $0.11387 \mathrm{~mm}$ wide and $1.5 \mathrm{~mm}$ long. Here, the patch is simulated using an iterative method. First, verified the convergence of the method (Figure $6(\mathrm{a})$ ). The patch must be $1.5 \mathrm{~mm}$ long and the excitation frequency is $\mathrm{f}=19.6 \mathrm{GHz}$. The results of frequency simulation have printed in the Figure $6(b)$.

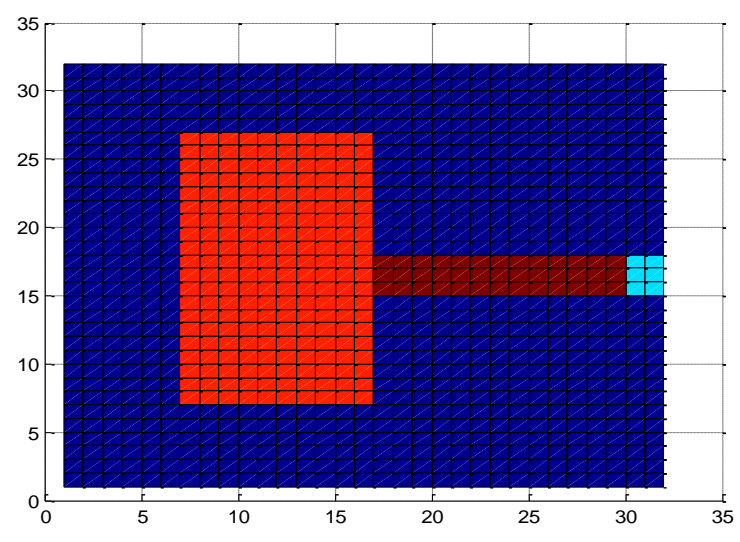

Figure 3. The electromagnetic structure 
The previous boundary conditions in the sub domains composites the dielectric interface have verified by the following three-dimensional curves
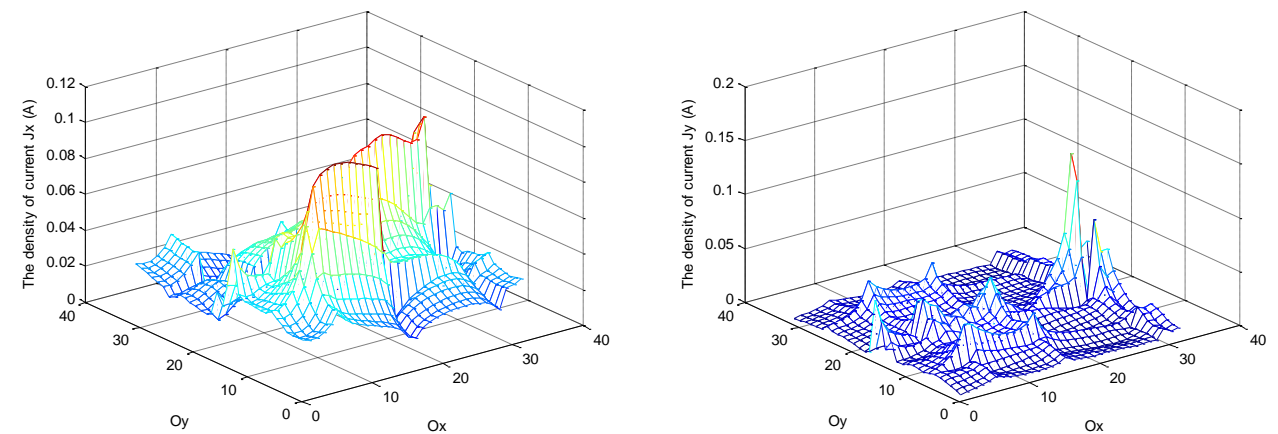

Figure 4. Current density Ji of patch antenna
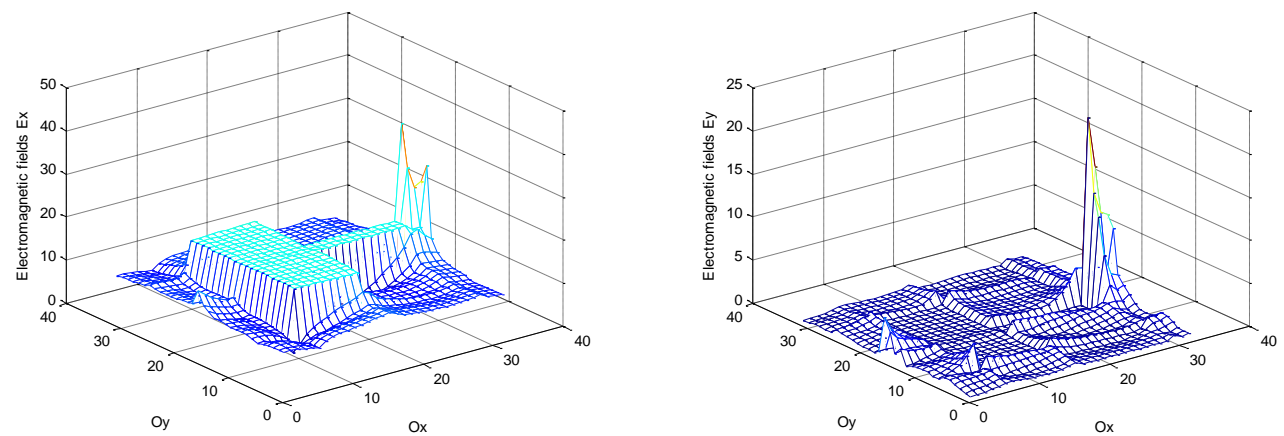

Figure 5. Electromagnetic fields Ei of patch antenna

The convergence test of the input impedance viewed by the source exciting is shown in Figure 6 (a). We see that, it is enough, approximately 144 iterations, to ensure the convergence of the iterative method WCIP.

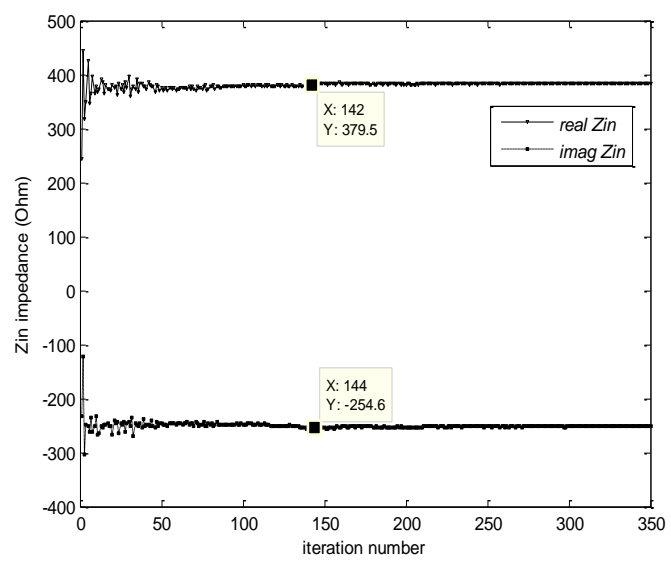

(a)

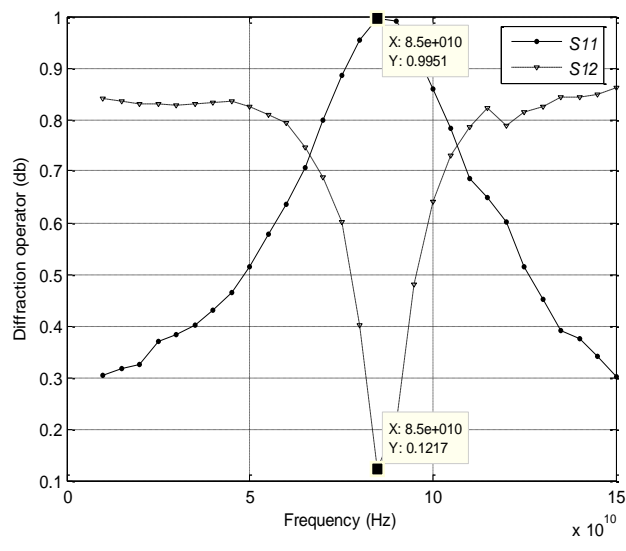

(b)

Figure 6. (a) Imaginary and real part of $Z_{\text {in }}$ as function of iteration number (b) Diffraction operator as function of frequency 


\subsection{Simulation of Multilayer Structure}

The second study is the electromagnetic multilayer structure, when the two dielectric interfaces consist of a patch antenna connected to a micro strip line section. The substrates characteristics are epsr $1=4.78$ with a thickness of $0.79 \mathrm{~mm}$. and epsr2=9.87 with a thickness of $0.5 \mathrm{~mm}$.

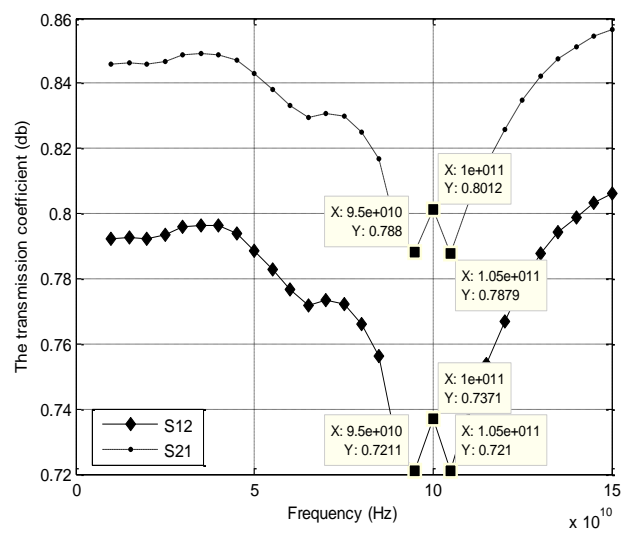

(a)

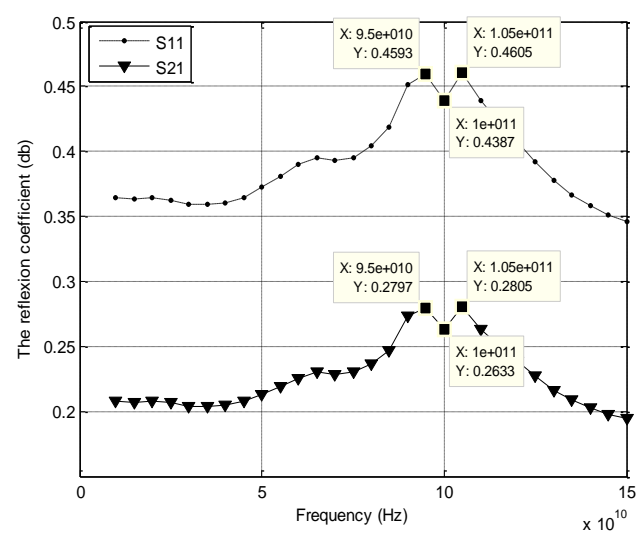

(b)

Figure 7. The diffraction operator as function of frequency (a) The transmmision coefficient (b) The reflection coefficient

- The resonance frequencies of electromagnetic structure as shown in the previous figure. Theses resonance frequencies are $9-10-10.5 \mathrm{GHz}$.

- The resonance box : $\mathrm{f}=4 \mathrm{GHz}$

- The resonance is periodic; it is repeated after $10 \mathrm{GHz}$.

- The wavelength is $30<\lambda_{\text {res }}<35 \mathrm{~mm}$

\section{Conclusion}

This paper is performed the transmission of electromagnetic waves on the multilayer antenna, and confirms that the WCIP method is also highly flexible and efficient for this type of circuits.

\section{References}

[1] Azizi (M), Aubert $(\mathrm{H})$, Bandrand $(\mathrm{H})$. A New Iterative Method for Scattering Problems. In European microwave conf., proc, 1995; 1: 255-258J.

[2] Gharsallah (A), Gharbi (A), Desclos (L), Baudrand (H), Analysis of Interdigital Capacitor and Quasilumped Miniaturized filters Using Iterative Method. Int. J. Numer. Mode I. 2002; 15: 169-179.

[3] Mami (A), Zairi $(H)$, Gharsallah (A), Baudrand $(H)$. Analysis of Micro-strip Spiral Induct or by Using Iterative Method, Microw. Opt. Technol. Lett. 2002; 35(4): 302-306,

[4] Gharsallah (A), Gharbi (A), Baudrand (H). Efficient Analysis of Multipon Pasrive Circuits Using the Iterative Technique Electromagnetics. Microw. Opt.Technol. Lett. 2001; 21: 75-84.

[5] Kaddour (M), Mami (A), Gharsallah (A), Gharbi (A), Baudrand (H). Analysis of Multilayer Microstrip Antennas by Using Iterative Method. Journal of Microwaves and Optoelectronics. April 2003; 3(1): 3952. 